\title{
Importance of individual and environmental variation for invasive species spread: a spatial integral projection model
}

\author{
Eelke Jongejans, ${ }^{1,6}$ Katriona Shea, ${ }^{2}$ Olav Skarpaas, ${ }^{3}$ Dave Kelly, ${ }^{4}$ and Stephen P. Ellner ${ }^{5}$ \\ ${ }^{1}$ Institute for Water and Wetland Research, Department of Experimental Plant Ecology, Radboud University Nijmegen, \\ Heyendaalseweg 135, 6525 AJ Nijmegen, The Netherlands \\ ${ }^{2}$ Pennsylvania State University, Department of Biology and IGDP in Ecology, 208 Mueller Laboratory, \\ University Park, Pennsylvania 16802 USA \\ ${ }^{3}$ Norwegian Institute for Nature Research, Gaustadalléen 21, NO-0349 Oslo, Norway \\ ${ }^{4}$ University of Canterbury, Biological Sciences, Private Bag 4800, Christchurch 8001 New Zealand \\ ${ }^{5}$ Cornell University, Department of Ecology and Evolutionary Biology, Ithaca, New York 14853 USA
}

Abstract. Plant survival, growth, and flowering are size dependent in many plant populations but also vary among individuals of the same size. This individual variation, along with variation in dispersal caused by differences in, e.g., seed release height, seed characteristics, and wind speed, is a key determinant of the spread rate of species through homogeneous landscapes. Here we develop spatial integral projection models (SIPMs) that include both demography and dispersal with continuous state variables. The advantage of this novel approach over discrete-stage spread models is that the effect of variation in plant size and size-dependent vital rates can be studied at much higher resolution. Comparing NeubertCaswell matrix models to SIPMs allowed us to assess the importance of including individual variation in the models. As a test case we parameterized a SIPM with previously published data on the invasive monocarpic thistle Carduus nutans in New Zealand. Spread rate $\left(c^{*}\right)$ estimates were $34 \%$ lower than for standard spatial matrix models and stabilized with as few as seven evenly distributed size classes. The SIPM allowed us to calculate spread rate elasticities over the range of plant sizes, showing the size range of seedlings that contributed most to $c^{*}$ through their survival, growth and reproduction. The annual transitions of these seedlings were also the most important ones for local population growth $(\lambda)$. However, seedlings that reproduced within a year contributed relatively more to $c^{*}$ than to $\lambda$. In contrast, plants that grow over several years to reach a large size and produce many more seeds, contributed relatively more to $\lambda$ than to $c^{*}$. We show that matrix models pick up some of these details, while other details disappear within wide size classes. Our results show that SIPMs integrate various sources of variation much better than discrete-stage matrix models. Simpler, heuristic models, however, remain very valuable in studies where the main goal is to investigate the general impact of a life history stage on population dynamics. We conclude with a discussion of future extensions of SIPMs, including incorporation of continuous time and environmental drivers.

Key words: Carduus nutans; integrodifference equation; invasions; matrix model; musk thistle; New Zealand; nodding thistle; spatial integral projection models (SIPMs); spatial population dynamics; Wald analytical long-distance model.

\section{INTRODUCTION}

A significant challenge in ecology is to accurately describe the ecological processes that cause changes in the distribution and abundance of organisms. This is particularly important when significant changes are occurring, such as invasive species that are increasing in spatial extent and local abundance, or endangered species that are declining spatially and numerically.

The utility of ecological population models for this goal is predicated on capturing essential features of the

Manuscript received 1 December 2009; revised 25 May 2010; accepted 28 May 2010; final version received 29 June 2010. Corresponding Editor: E. E. Crone.

6E-mail: E.Jongejans@science.ru.nl population biology of the species in question, including both demography and dispersal. Models for the spatial dynamics of unstructured populations have a long pedigree (Skellam 1951, Okubo et al. 2001). Recent advances, linking matrix population models with integrodifference equations (Neubert-Caswell models [NCMs]; Neubert and Caswell 2000, Lewis et al. 2006), have greatly improved our understanding of how local demographic processes can affect spatial spread, through the development of measures for the sensitivity and elasticity of wave speeds to demographic parameters. Unfortunately, however, matrix models require the imposition of discrete categories even if such categories do not exist (Caswell 2001). For example, insects, with their discrete developmental stages, are 
well described by such structured population models. However, many species exhibit continuous distributions of important characteristics, such as continuous size distributions, that do not conform easily to categorization (Fig. 1). The position of category boundaries can make large differences to predictions (Enright et al. 1995). Vital rates (i.e., survival, growth, and reproduction rates) of small individuals within a particular stage class can have distinctly different means and variances than large individuals in that same stage class. Although attempts to include variation around means in each category have been made (Zuidema and Franco 2001), individual variation is still commonly ignored. However, ignoring the individual variation within these artificial categories can lead to quantitative and even qualitative differences in predictions (Easterling et al. 2000, Pfister and Stevens 2003, Morris and Doak 2004, Shea et al. 2006). Individual-based models (IBMs) allow individuals to be tracked, but sacrifice analytic results for simulation methods (DeAngelis and Gross 1992, Judson 1994). Thus, both of the commonly used methods, matrix models and IBMs, are unsatisfactory in the face of individual variation. Because individual variation is common, this is a serious handicap.

Recently, a new approach has been developed that unites a flexible framework for modeling multiple sources of variance with the technical arsenal of tools developed for matrix models in an analytic framework: integral projection models (IPMs; Easterling et al. 2000, Ellner and Rees 2006). Instead of discretizing continuous-state variables into representative categories, the continuous function itself is used in the model. This technique is rapidly gaining in popularity because of its obviously utility (Godfray and Rees 2002, Metcalf et al. 2003, Rose et al. 2005, Williams and Crone 2006, Kuss et al. 2008, Zuidema et al. 2010). Reanalysis of data previously used in matrix models provides additional ecological insights (e.g., Easterling et al. 2000) and has greatly improved our understanding of how complex local demographic processes, and the associated individual variation, affect population growth and the evolution of life history strategies (Rees and Rose 2002, Childs et al. 2003, Ellner and Rees 2006).

Here we take the logical and important next step of developing spatial integral projection model (SIPMs). By combining the analytical methods for modeling structured demography (IPM) with the analytical methods for modeling spatial spread (NCMs) in discrete time, we can obtain detailed projections of population growth and spread, as well as sensitivities, elasticities, and variance decomposition (life table response experiments; LTREs) for spatial spread as well as for population growth rates. These are all measures that are important in the analysis of spatiotemporal dynamics of species, and are critical in studies of species that require management. We anticipate that the increased resolution and detail that can be obtained, backed by analytical power, will allow better understanding of the factors affecting such problem species, and will hence lead to improved management. As a case study we analyze the dynamics of a SIPM for the invasion of the nodding thistle, Carduus nutans, in New Zealand.

\section{Material And Methods}

Study system

Carduus nutans L. (Asteraceae; see Plate 1), musk or nodding thistle, is a Eurasian rangeland weed of major economic importance in the United States (Skinner et al. 2000), southern Africa, Australia (Jongejans et al. 2008), and New Zealand, our study site (Shea and Kelly 1998). C. nutans is a short-lived monocarpic perennial (plants die after reproduction). Seeds typically germinate in autumn and grow as rosettes for six months to three years before flowering, which is dependent on vernalization and on individual age and size (Popay and Medd 1990). Flowering plants are $20-200 \mathrm{~cm}$ tall, with numerous solitary flower heads $1.5-4.5 \mathrm{~cm}$ in diameter. C. nutans flowers from summer to early fall (DecemberMarch in New Zealand). Many wind-dispersed seeds can be produced per individual. Fresh seed germinates well, but may survive for more than a decade in undisturbed soil (Burnside et al. 1981).

We used demographic data collected along transects in a sheep- and cattle-grazed pasture near Midland, New Zealand between 1985 and 1988 (for details see Shea and Kelly 1998). At this site, about $40 \%$ of flowering plants were in their first year (annuals), 55\% were two years old (biennials), and $4 \%$ were three years old (Kelly and McCallum 1990). The natural log of rosette area $\left(\mathrm{cm}^{2}\right)$ is a good predictor for C. nutans vital rates (Shea and Kelly 1998, Rees et al. 2006). Here we reanalyzed how survival and flowering were related to $\ln$ (rosette area) (for simplicity: "size") by using a binomial regression model (glmmML: generalized linear model with random intercept) in which transect was included as a random, clustering factor. Next-year's size (mean and variation) of surviving plants was analyzed with a linear regression model (gls, generalized least square) with this year's plant size as an explanatory variable. Seedling sizes were fitted with a normal distribution. The number of flower heads per flowering plant was analyzed with a linear model, using rosette area as an explanatory variable. Data from different calendar years were pooled to improve statistical power. These statistical analyses and all subsequent model analyses were performed in $\mathrm{R}(\mathrm{R}$ Development Core Team 2009). This statistical approach used for fitting models to life history data is exactly as for an individual-based model (IBM; e.g., Shea et al. [2006] for an example of C. nutans in Australia), but with the advantage that the IPM is analytic.

\section{Notation}

We are melding two bodies of theory; unfortunately, they frequently use the same symbols to mean different things. To avoid confusion, we largely retain the 

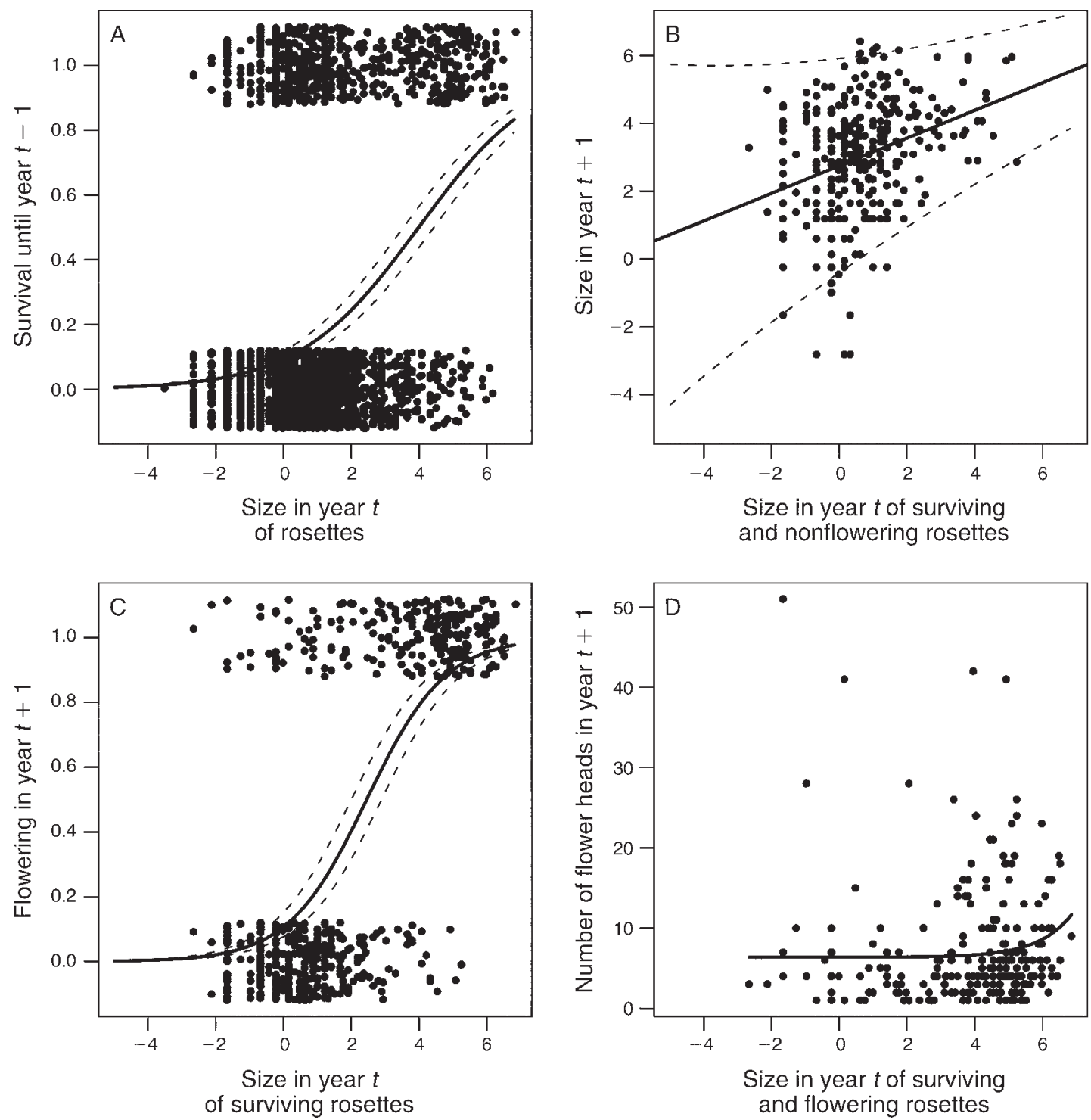

FIG. 1. Parameterization of vital rate functions in the thistle Carduus nutans (dashed lines are $95 \%$ CIs) in relation to rosette "size" (natural-log-transformed rosette area, originally measured in $\left.\mathrm{cm}^{2}\right)$. (A) Survival until the next summer of year $t+1: \ln [\sigma /(1-$ $\sigma)]=-2.270(\mathrm{SE}=0.095)+0.569(\mathrm{SE}=0.026) z_{1} ; n=4103, P<0.001$. (B) Annual growth of surviving, nonflowering rosettes: $z_{2}=$ $2.751(\mathrm{SE}=0.097)+0.407(\mathrm{SE}=0.55) z_{1} ; \mathrm{SD}=\left[9.119 \exp \left(-0.228 \times 2 z_{1}\right)\right]^{0.5} ; n=342, P<0.001$. (C) The probability that next year's surviving rosettes will flower: $\ln [\beta /(1-\beta)]=-2.107(\mathrm{SE}=0.210)+0.860(\mathrm{SE}=0.066) z_{1} ; n=578, P<0.001$. (D) The number of flower heads of surviving and flowering rosettes in the next summer: $\omega=6.363+0.0056 \exp \left(z_{1}\right) ; n=236, P<0.001$ (using Kendall's tau to test for correlation between $z_{1}$ and $\omega$ ).

symbols commonly used in the larger, long-standing dispersal and spread literature, and make some modifications to the notation that has been used for IPMs to date (see Table 1).

\section{Integral Projection Models (for demographic processes)}

An IPM is a discrete-time model that describes changes in a population structured by one or more continuous individual-level state variables, and possibly also by one or more discrete individual-level state variables. Any continuous state variable can be used, but we here focus on individual size. Following Easterling et al. (2000) we can describe the population by the number of rosettes of size $z_{1}$ at time $t, R\left(z_{1}, t\right)$. One time step later, the number of rosettes of size $z_{2}$ is then given by

$$
R\left(z_{2}, t+1\right)=\int_{z} K_{\mathrm{dem}}\left(z_{2}, z_{1}\right) R\left(z_{1}, t\right) d z_{1}
$$

where the integration is over the set of all possible sizes, $Z$, and where the demographic kernel $K_{\text {dem }}$, describes all the ways in which rosettes of size $z_{1}$ at time $t$ can 
TABLE 1. Key to notation used in the spatial integral projection models (SIPMs) for an invasive thistle. Carduus nutans, in New Zealand.

\begin{tabular}{|c|c|c|}
\hline Symbol & Definition & New Zealand data \\
\hline $\mathrm{Z}$ & set of all possible rosette sizes & \\
\hline$z_{1}, z_{2}$ & $\begin{array}{l}\text { rosette size [ln rosette area] of an individual at years } t \\
\text { and } t+1 \text {, respectively }\end{array}$ & mean $\left(z_{1}\right)=0.321, \mathrm{SD}\left(z_{1}\right)=1.594$ \\
\hline$X$ & set of all possible locations & \\
\hline$x_{1}, x_{2}$ & $\begin{array}{l}\text { location in one dimension of an individual at } t \text { and } t+ \\
1 \text {, respectively }\end{array}$ & \\
\hline$n, \mathbf{n}$ & population size (as scalar or vector, respectively) & \\
\hline$R(z)$ & the number of rosettes of size $z$ & \\
\hline$B$ & the number of seeds in the seed bank & \\
\hline$K_{\mathrm{dem}}$ & $\begin{array}{l}\text { demographic kernel (comprises rosette survival, } \\
\text { growth, and reproduction) }\end{array}$ & \\
\hline$Q$ & fecundity component of the demographic model & \\
\hline & survival/growth component of the demographic model & \\
\hline$\sigma\left(z_{1}\right)$ & rosette survival probability & $\begin{array}{c}\ln (\sigma /(1-\sigma))=-2.270(\mathrm{SE}=0.095)+0.569(\mathrm{SE}= \\
0.026) z_{1} ; \mathrm{SD}=0.129 ; n=4103 ; P<0.001\end{array}$ \\
\hline$\gamma\left(z_{2}, z_{1}\right)$ & growth function of surviving, nonflowering rosettes & $\begin{array}{l}z_{2}=2.751(\mathrm{SE}=0.097)+0.407(\mathrm{SE}=0.55) z_{1} ; \mathrm{SD}= \\
\quad\left(9.119 \exp \left(-0.228 \times 2 z_{1}\right)\right)^{0.5} ; n=342 ; P<0.001\end{array}$ \\
\hline$\beta\left(z_{1}\right)$ & flowering probability of surviving rosettes & $\begin{array}{c}\ln (\beta /(1-\beta))=-2.107(\mathrm{SE}=0.210)+0.860(\mathrm{SE}= \\
0.066) z_{1} ; \mathrm{SD}=0.196 ; n=578 ; P<0.001\end{array}$ \\
\hline$\omega\left(z_{1}\right)$ & number of flower heads per flowering rosette & $\omega=6.363+0.0056 \exp \left(z_{1}\right) ; n=236, P<0.001$ \\
\hline & potential seed production per flower head & $\pi=374$ \\
\hline$\phi$ & probability that a potential seed escapes florivory & $\phi=1$ \\
\hline & probability that a new seed establishes as a seedling & $\varepsilon=0.019$ \\
\hline$\chi\left(z_{2}\right)$ & distribution of seedling sizes & mean $=-0.771, \mathrm{SD}=1.719$ \\
\hline $\mathrm{v}$ & probability that a new seed enters the seed bank & $v=0.157$ \\
\hline$\sigma_{1}$ & $\begin{array}{l}\text { probability of seed survival and retention in the seed } \\
\text { bank }\end{array}$ & $\sigma_{1}=0.038$ \\
\hline$\varepsilon_{1}$ & $\begin{array}{l}\text { probability that a seed in the seed bank survives and } \\
\text { establishes as a seedling }\end{array}$ & $\varepsilon_{1}=0.1847$ \\
\hline$\lambda$ & projected population growth rate & $\lambda=1.88$ for SIPM 100 \\
\hline$K_{\text {disp }}$ & dispersal kernel & \\
\hline$k(r)$ & $\begin{array}{l}\text { Wald (inverse Gaussian) probability density function } \\
\text { for dispersal distances } r\end{array}$ & \\
\hline$\mu, \tau$ & $\begin{array}{l}\text { mean (location parameter) and scale parameter in the } \\
\text { Wald model }\end{array}$ & \\
\hline$F$ & terminal velocity $(\mathrm{m} / \mathrm{s})$ & \\
\hline$L, h$ & $\begin{array}{l}\text { seed release height and surrounding vegetation height } \\
(\mathrm{m})\end{array}$ & \\
\hline$U$ & wind speed at $10 \mathrm{~m}$ above ground $(\mathrm{m} / \mathrm{s})$ & \\
\hline$M(j)$ & moment-generating function & \\
\hline $\mathbf{H}_{s}$ & $\begin{array}{l}\text { matrix of which the elements are values of the } \\
\text { moment-generating function for the spatial } \\
\text { distribution of type- } z_{2} \text { individuals at time } t+1 \\
\text { produced by type- } z_{1} \text { individuals at time } t\end{array}$ & \\
\hline$\delta_{0}$ & $\begin{array}{l}\text { the Dirac delta function, i.e., a probability distribution } \\
\text { with all mass at } 0\end{array}$ & \\
\hline$c$ & invasion speed $(\mathrm{m} / \mathrm{yr}) ; c^{*}$ is the asymptotic spread rate & $c^{*}=13.9$ for SIPM 100 \\
\hline & invasion shape parameter $\left(s^{*}\right.$ corresponds to $\left.c^{*}\right)$ & \\
\hline elas & elasticity of $\lambda$ or $c^{*}$ to changes in $\mathbf{A}, K_{\mathrm{dem}}$ or $K_{\mathrm{disp}}$ & \\
\hline
\end{tabular}

contribute to the number of rosettes of size $z_{2}$ at time $t+$ 1. Here we write out the vital rates that underlie $K_{\mathrm{dem}}$ (consistent with the vital rates used in an autumn-toautumn transition matrix model for $C$. nutans by Jongejans et al. (2008)):

$K_{\mathrm{dem}}=\sigma\left(z_{1}\right)\left[\left(1-\beta\left(z_{1}\right)\right) \gamma\left(z_{2}, z_{1}\right)+\beta\left(z_{1}\right) \omega\left(z_{1}\right) \pi \phi \varepsilon \chi\left(z_{2}\right)\right]$.

Thus $K_{\text {dem }}$ includes individuals that survive until the next summer, $\sigma\left(z_{1}\right)$, and then either remain as rosettes, 1 $-\beta\left(z_{1}\right)$, and grow to their new size, $\gamma\left(z_{2}, z_{1}\right)$, as well as survivors that flower, $\beta\left(z_{1}\right)$, and produce new individuals, $\omega\left(z_{1}\right) \pi \phi \varepsilon \chi\left(z_{2}\right)$. This latter fecundity function has the following components: $\omega\left(z_{1}\right)$ is a size-dependent poten- tial flower head production term, $\pi$ is the average number of potential seeds per flower head, $\phi$ is the probability that a potential seed escapes florivory and becomes a seed, $\varepsilon$ is the chance that a seed establishes as a seedling, and $\chi\left(z_{2}\right)$ is the probability density function of a seedling growing to a certain size by the time of the next census. The existence of a seed bank requires the addition of a separate discrete-state variable (Ellner and Rees 2006) that is linked to $K_{\text {dem }}$ by the proportion of newly produced seeds that enter the seed bank, $v$, and the proportion of seeds in the seed bank that survive and establish to become seedlings, $\varepsilon_{1}$. A proportion, $\sigma_{1}$, of the seeds in the seed bank survives and stays there. The combined seed bank $(B)$ and rosette $(R)$ IPM thus 
becomes

$$
B(t+1)=\sigma_{1} B(t)+\int \beta\left(z_{1}\right) \omega\left(z_{1}\right) \pi \phi \vee R\left(z_{1}, t\right) d z_{1}
$$

and

$$
R\left(z_{2}, t+1\right)=\varepsilon \chi\left(z_{2}\right) B(t)+\int K_{\mathrm{dem}}\left(z_{2}, z_{1}\right) R\left(z_{1}, t\right) d z_{1} .
$$

Eq. 3 shows that seeds in the seed bank at time $t+1$ come from two sources: seeds at time $t$ that survived but did not germinate, and rosettes at time $t$ that produced seeds that entered the seed bank. For the New Zealand population, $\chi\left(z_{2}\right)$ had a mean of -0.771 and a variance of $1.719, \pi=374, \phi=1, v=0.157, \varepsilon=0.0194$, and $\varepsilon_{1}=$ 0.1847 (for details, see Jongejans et al. 2008). Data on the size-dependent vital rates are shown in Fig. 1.

\section{Dispersal kernels (for individual movement)}

The models that we develop for spatial population spread require a dispersal kernel $k(r)$, which is the probability density function for seed dispersal distances $r$ (in $C$. nutans this is the only mode of reproduction and the only stage at which dispersal occurs). We use the Wald analytical long-distance dispersal (WALD) model (Katul et al. 2005). This model is derived from wellestablished models in fluid mechanics and predicts an inverse Gaussian (Wald) distribution of dispersal distances $r$ (Evans et al. 1993, Katul et al. 2005):

$$
k(r)=\left(\frac{\tau}{2 \pi r^{3}}\right)^{1 / 2} \exp \left(-\frac{\tau(r-\mu)^{2}}{2 \mu^{2} r}\right)
$$

where $\mu$ is the mean (the location parameter) and $\tau$ is the scale parameter. In contrast to empirical (or phenomenological) models of dispersal (e.g., exponential), WALD has the desirable property that its parameters are directly linked to characteristics of the species and the environment: $\mu=L U / F$ and $\tau=(L / \psi)^{2}$, where $L$ is the seed release height, $F$ is terminal falling velocity of seeds, $U$ is the horizontal wind velocity between $L$ and the ground, and $\psi$ is a turbulent flow parameter that reflects wind variation (Katul et al. 2005). Furthermore, the WALD model (with a natural distribution of hourly means of $U$ to integrate across the dispersal season) has been shown to perform at least as well as a range of commonly used empirical dispersal models for C. nutans (Skarpaas and Shea 2007). We used the same New Zealand dispersal parameters as Jongejans et al. (2008).

\section{Linking IPMs with dispersal to model spatial spread}

To link dispersal and population spread, we used the framework of integrodifference equations (shown in detail in the Appendix). This framework can accommodate a wide range of population models and dispersal kernels, unlike, for instance, the diffusion approach (Skellam 1951, Okubo et al. 2001). Details on how to estimate population wave speeds can be found in
Neubert and Caswell (2000), Neubert and Parker (2004), and Lewis et al. (2006); relevant points are summarized here. The general formulation of the Neubert-Caswell Model (NCM) is

$$
\mathbf{n}\left(x_{2}, z_{2}, t+1\right)=\iint \tilde{K}\left(x_{2}, x_{1}, z_{2}, z_{1}\right) \mathbf{n}\left(x_{1}, z_{1}, t\right) d x_{1} d z_{1}
$$

where $\mathbf{n}\left(x_{1}, z_{1}, t\right)$ and $\mathbf{n}\left(x_{2}, z_{2}, t+1\right)$ are the structured population stage vectors (i.e., the population density in each stage, including both $B$ and $R$ ) at location $x_{1}$ at time $t$ and at location $x_{2}$ one time step later. The integration is over the set of all possible locations and size categories. $\tilde{K}\left(x_{2}, x_{1}, z_{2}, z_{1}\right)$ is the combined demography and dispersal kernel. In the original NCM, the demography is described by a matrix projection model with discrete size categories; here, the demography is described by the demographic model; see Eqs. 3 and 4). In short, the model expresses the population structure and density at location $x_{2}$ at time $t+1$ as a sum of inputs (through survival, reproduction, and dispersal) from all locations $x_{1}$ at time $t$.

As can be seen from Eqs. 1 and 6, spatial and nonspatial integral projection models have the same formulation: location is just an additional way for a population to be structured. The combination of the two allows us to explore in great detail how demographic and dispersal components differentially contribute to the spatial spread of species. The models make more efficient use of data (especially when data are relatively sparse) and require fewer fitted parameters than would a conventional matrix model of the required complexity (Ellner and Rees 2006). Sensitivities and elasticities are not tied to the stage categories that have to be chosen for matrix models, but are calculated over the continuum of plant sizes.

Comparing NCMs with Spatial Integral Projection Models (SIPMs) allowed us to assess the importance of including individual variation in the models. To do so we evaluated the SIPMs with different-sized matrices. Thus, "SIPM100" indicates an SIPM that is implemented using a $100 \times 100$ matrix to evaluate integrals, using the numerical methods of Ellner and Rees (2006).

\section{Projecting rates of population spread}

In a homogeneous environment, dispersal only depends on the relative locations of the points where movement initiates and finishes. Under the assumption of spatial homogeneity (i.e., that vital rates do not depend on location), the model can be simplified by considering its long-term behavior. For monotonic (such as exponential or logistic) population growth rates larger than unity and for thin-tailed (exponentially bounded) dispersal kernels, the population spread rate converges to a constant speed, $c^{*}$, (Lewis et al. 2006):

$$
c^{*}=\min _{s>0}\left[\frac{1}{s} \log \left(\rho_{s}\right)\right]
$$


where $\rho_{s}$ is the dominant eigenvalue of $H_{s}$ (see Eq. 9); $s$ is an auxiliary variable that is a measure of the steepness of the spreading population wave. In the Appendix, we generalize these results to general SIPMs (Eq. 6) and discuss the conditions required for Eq. 7 to be valid.

For species such as $C$. nutans in which new seeds disperse but all other life stages are sessile, the combined demography-dispersal kernel will typically have the following form:

$$
\begin{aligned}
\tilde{K}\left(x_{2}, x_{1}, z_{2}, z_{1}\right)= & K_{\text {disp }}\left(x_{2}-x_{1}\right) Q\left(z_{2}, z_{1}\right) \\
& +\delta_{0}\left(x_{2}-x_{1}\right) G\left(z_{2}, z_{1}\right)
\end{aligned}
$$

where $K_{\text {disp }}$ is the dispersal kernel, $Q$ and $G$ are, respectively, the fecundity and survival/growth components of the demographic model (Eqs. 3 and 4 ), and $\delta_{0}$ is the Dirac delta function, i.e., a probability distribution with all mass at 0 (no movement). For kernels of the form in Eq. 8, which apply whenever all dispersing life stages have the same dispersal pattern, Eq. A8 (Appendix) shows that $\rho_{s}$ is the dominant eigenvalue of the kernel

$$
H_{s}\left(z_{2}, z_{1}\right)=M(s) Q\left(z_{2}, z_{1}\right)+G\left(z_{2}, z_{1}\right)
$$

where $M(s)$ is the moment-generating function of the one-dimensional dispersal kernel. The general definition of $\mathbf{H}_{s}$, applicable to any SIPM, is given in the Appendix: Eq. A8.

The WALD model describes radial dispersal distances. To calculate the one-dimensional moment-generating function for Eq. 9, we used the empirical momentgenerating function approach described in Skarpaas and Shea (2007). We first simulated radial dispersal distances with the WALD model in random directions, assuming a lognormal seasonal distribution of hourly mean wind speeds, with mean and standard deviation estimated from local wind measurements (Jongejans et al. 2008). We then marginalized these simulated dispersal vectors onto one spatial axis (Lewis et al. 2006). These marginalized dispersal distances were then used for calculating the empirical moment-generating function.

\section{$c^{*}$ elasticities}

Perturbation analyses show how sensitive model outputs are to changes in model parameters. Elasticities measure the relative effect on $\lambda$ by a relative change in an element $a_{i j}$ : elas $\left(a_{i j}\right)=\left(a_{i j} / \lambda\right)\left(\delta \lambda / \delta a_{i j}\right)$ (de Kroon et al. 2000). Because the elasticity values of a matrix sum to unity, they can be regarded as the proportional contributions made by the respective matrix elements to $\lambda$. In IPMs, elasticity of $\lambda$ measures the relative rate of increase in $\lambda$ when a small disk centered around a point on the kernel is raised by a small percentage (Easterling et al. 2000, Ellner and Rees 2006). The elasticity function elas $\left(z_{1}, z_{2}\right)$ defined this way integrates to unity, so it can also be regarded as the proportional contribution to $\lambda$.
In matrix models, the sensitivity (and elasticity) of the invasion speed, $c^{*}$, to changes in the matrix elements and dispersal parameters can be calculated in a similar way as $\lambda$ elasticities (Eqs. 26-29 in Neubert and Caswell 2000, Shea 2004). In the Appendix, we generalize these results to SIPMs. The sensitivity of $c^{*}$ to changes in any model parameter $\theta$ or any component of the kernel is given by

$$
\frac{\partial c^{*}}{\partial \theta}=\left.\left[\frac{1}{s \rho_{1}} \frac{\partial \rho_{1}}{\partial \theta}\right]\right|_{s=s^{*}}
$$

Because $\rho_{1}$ is the dominant eigenvalue of the kernel $\mathbf{H}_{s}, \partial \rho_{1} / \partial \theta$ can be computed (using the sensitivity formula for IPMs) as $\left\langle v_{s},\left(\partial \mathbf{H}_{s} / \partial \theta\right) w_{s}\right\rangle /\left\langle v_{s}, w_{s}\right\rangle$, where $v_{s}, w_{s}$ are the dominant left and right eigenvectors of $\mathbf{H}_{s}$. The elasticity to the different parameters of the dispersal kernel $K_{\text {disp }}$ is particularly interesting because it provides researchers with an analytical tool to study the contributions of dispersal parameters to the spread rate, relative to contributions of demographic parameters (Jongejans et al. 2008). For example, the elasticity for terminal velocity can be determined from the definition

$$
\operatorname{elas}(F)=\frac{F}{c^{*}} \frac{\partial c^{*}}{\partial F}
$$

in combination with Eq. 10.

\section{Results}

Our analysis of the demographic data from the New Zealand Midland population showed that survival, growth, flowering, and flower-head production rates varied with plant size (Fig. 1). The effect of different stage boundaries in matrix models becomes apparent in Fig. 2, where we plot the projected total population growth rate $(\lambda$, panel $\mathrm{A})$ and invasion speed $\left(c^{*}\right.$, panel B) calculated with the same SIPM but with increasing numbers of stage class boundaries imposed on the range of plant sizes. SIPMs with small numbers of stage classes show large fluctuations, but $\lambda$ and $c^{*}$ stabilize from approximately seven size classes upward. The $\lambda$ values of SIPM100 and IPM100 were identical (1.88), because $\lambda$ is the growth rate of the entire population, ignoring space. Lumping plants with a wide range of sizes into the same stage class has similar ramifications for the stable stage distributions at the invasion front, as projected by models with different number of classes (Fig. 2C). An SIPM100, for instance, has a much smoother stable size distribution than an SIPM4 or the four-class matrix model used previously (e.g., by Shea and Kelly 1998, Jongejans et al. 2008). These findings reinforce that IPMs and SIPMs incorporate the continuous nature of many vital rate-plant trait relationships well.

The invasion speed $\left(c^{*}=13.9 \mathrm{~m} / \mathrm{yr}\right)$ projected by the SIPM100 is sensitive not only to the variation in vital rates between plants of different size (Fig. 2B), but also to variation in the size of new seedlings and to variation in the growth of equal-sized survivors. This can be seen 



FIG. 2. Impact of the dimension of a spatial integral projection model (SIPM) on (A) the projected total population growth rate $(\lambda)$, on (B) the projected invasion wave speed $\left(c^{*}\right)$, and on $(\mathrm{C})$ the stable stage distribution (SSD) of the invasion wave front population of Carduus nutans in relation to rosette size (natural $\log$ of rosette area). The SIPM dimension gives the size of the matrix (number of imposed subdivisions of the continuous variable) used to implement the model numerically, in order to project population growth or spread. The black square in panels (A) and (B) indicates the $\lambda$ and $c^{*}$ values of the previously published $4 \times 4$ matrix model (NCM4). In panel (C), a SIPM100 is compared with a SIPM4 and the NCM4. Two dots at 4.4 and 5.2 on the $x$-axis indicate the borders between the small, medium-sized, and large rosette size classes in the NCM4 (as clearly seen in Fig. 4). by artificially reducing the variance of the seedling sizes to $1 \%$ of its original value: $c^{*}$ is then reduced to $8.4 \mathrm{~m} /$ yr. When the variance around the mean growth function is reduced to $1 \%, c^{*}$ decreases to $12.8 \mathrm{~m} / \mathrm{yr}$. Other sources of variation are important, too: setting the standard deviation of the ln-transformed terminal velocity values to $1 \%$ reduces $c^{*}$ to $12.9 \mathrm{~m} / \mathrm{yr}$, whereas reducing the standard deviation of the ln-transformed wind speeds to $1 \%$ brings $c^{*}$ down as low as $2.5 \mathrm{~m} / \mathrm{yr}$. The large impacts of variation in seedling size and wind speed imply that extreme events set the pace of population spread.

A more structured way of studying the dynamics of the SIPM100 is to look at the elasticity of $c^{*}$ to model parameters. The vital rate $c^{*}$ elasticity values are mostly similar to the $\lambda$ elasticity values of these vital rates (Fig. 3 ): both the local population growth rate and the invasion speed are particularly sensitive to proportional change in survival and sexual reproduction parameters. The elasticity landscape of the SIPM100 shows three main regions of high contribution to $c^{*}$ (Fig. 4A): the highest density of elasticity values involves small rosettes (sizes between $\sim 0$ and 2 (ln of rosette area, measured in $\left.\mathrm{cm}^{2}\right)$ ) that produce new, small rosettes next year through a reproduction self-loop (i.e., behaving as annual plants). A two-year loop can be seen as well: high elasticity values are associated with seedlings that survive as nonflowering rosettes until the next year and grow to a size of $\sim 4$. This elasticity peak is matched partly with high elasticity values for similarly sized $(\sim 4)$ rosettes that each produce many new seedlings.

To clarify the differences between the $c^{*}$ contributions of survival and reproduction, we integrated the elasticity values over the initial plant sizes for these two life cycle components separately (Fig. 4B). Similarly, elasticity values integrated over next year's size summarize the $c^{*}$ contributions of surviving rosettes and new seedlings (Fig. 4C). Importantly, these different elasticity peaks all occur within the size range of the "small" stage in the $4 \times 4$ matrix models. The $\lambda$ elasticity landscape of the IPM100 has the same peaks as the $c^{*}$ elasticity landscape in Fig. 4A, but comparing them in detail shows that the small annual plants (seedling-to-seedling self-loop) are even more important for $c^{*}$, whereas the survival and growth of seedling-sized to larger rosettes contributes less to $c^{*}$ than to $\lambda$ (Fig. 4D).

\section{Discussion}

The spatial integral projection model (SIPM) for Carduus nutans is a novel combination of integral projection models (IPM) with Neubert-Caswell integrodifference models for invasion speeds of structured populations (NCM). This combination of two theoretical approaches (Easterling et al. 2000, Neubert and Caswell 2000) allows spatial spread to be modeled with effectively continuous life history variation in an analytic framework for the first time. This leads to an improved understanding of how variation in demogra- 




FIG. 3. Elasticity values of population growth rate ( $\lambda$, black bars) and invasion wave speed ( $c^{*}$, gray bars) for demography and dispersal rates in Carduus nutans. Elasticity values for $\lambda$ were rescaled by dividing each by the sum (5.6) of the absolute values of the $\lambda$ elasticity values. Similarly, all $c^{*}$ elasticity values were rescaled by dividing them by the sum (8.4) of the absolute values of the demographic rates' $c^{*}$ elasticity values. This rescaling allows for comparison of the relative importance of a demographic vital rate for $\lambda$ and $c^{*}$, and for comparison among the $c^{*}$ elasticity values of demography and dispersal rates. Parameters of the linear models include intercept, size-slope (the slope with respect to the size axis, $z_{1}$ ), and in the case of growth, also variance and size-variance.

phy and dispersal rates differentially contribute to spatial spread of species.

\section{SIPMs deal with variation much better...}

IPMs and SIPMs are important steps in the direction of including multiple types and sources of variation in analytical models. The example of the invasive thistle Carduus nutans in New Zealand shows that individual variation can be substantive: the size of the rosettes of this species can vary over three orders of magnitude, which has a substantial impact on whether these plants will flower, on how large they will be in the next year if they do not flower and/or die, and their chance of dying (Fig. 1). Furthermore, growth can also vary greatly among individuals of the same size. Matrix models include this size and growth variation only roughly; ignoring or simplifying variation too much can quickly result in large changes in projection of the population growth rate $\lambda$ (Fig. 2A), wave speed $c^{*}$ (Fig. 2B), and stable stage distributions at the front of an invasion (Fig. 2C).

Analyzing Aconitum noveborancense data, Easterling et al. (2000) found that elasticity surfaces picked up the details of the plant's life history far better than the counterpart matrix model. We show that elasticity surface plots are also useful when analyzing which life cycle components contribute most to spatial spread (Fig. 4A), especially when combined with integrations over plant size at the beginning (Fig. 4B) and end (Fig. 4C) of a year. For the New Zealand $C$. nutans case, the $c^{*}$ elasticity analysis pointed out which plants contributed most to $c^{*}$ through survival (the plants in the -2 to 3 size range, i.e., rosette areas of $0.1-20 \mathrm{~cm}^{2}$ ) and which plants contributed most through sexual reproduction (plants of sizes 0 to 7, with a peak between 1 and 2, i.e., rosette area $3-7 \mathrm{~cm}^{2}$ ). Such details clearly would have been lost 

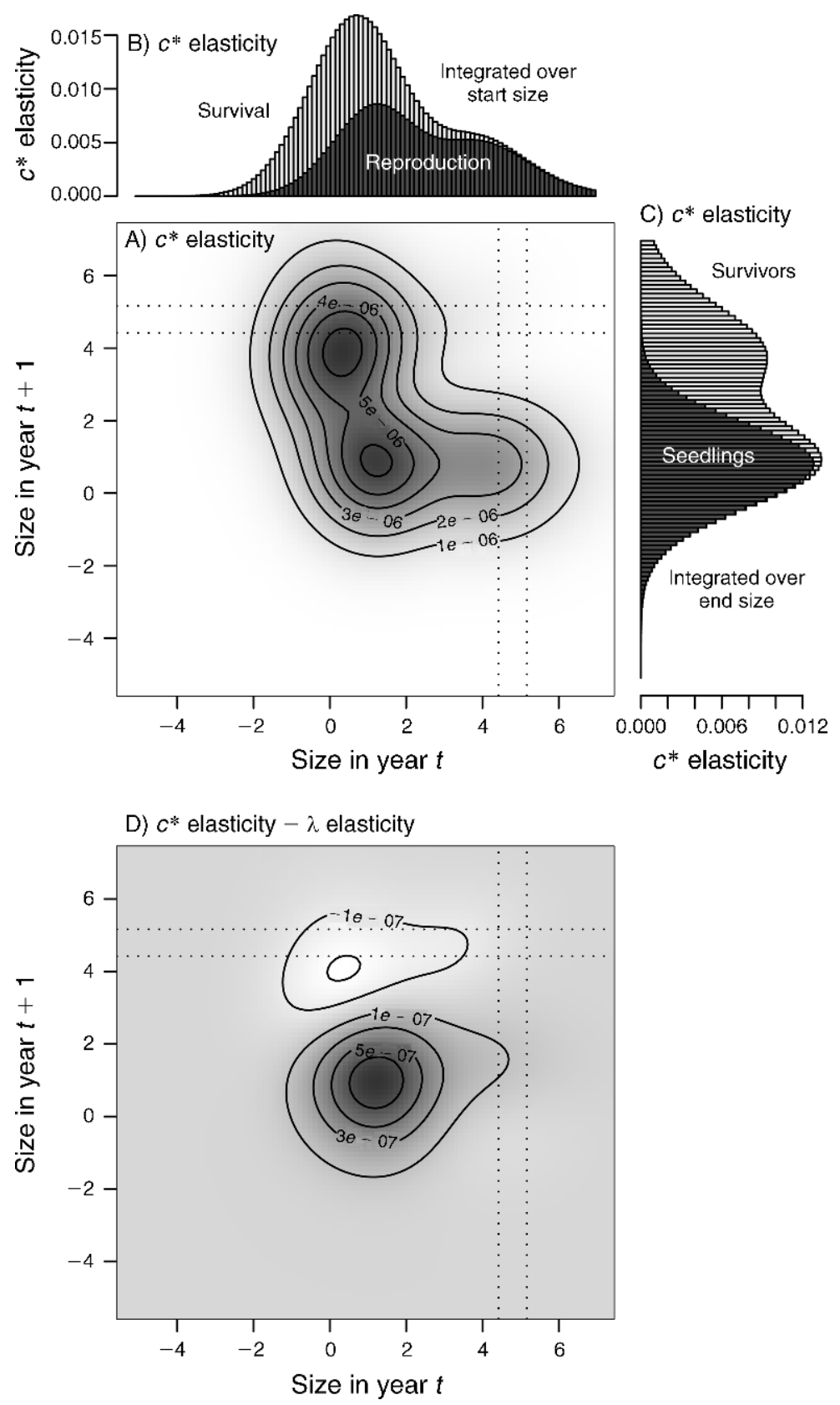

FIG. 4. Elasticity function (A) of invasion speed $\left(c^{*}\right)$ of Carduus nutans projected with a SIPM100, showing the relative change in $c^{*}$ with proportional changes in the demography kernel, $\left(K_{\mathrm{dem}}\right)$, which gives the transition (through survival and growth) and contribution (through reproduction) probabilities for each combination of plant size in year $t\left(z_{1}, x\right.$-axis) and plant size in year $t+1$ $\left(z_{2}, y\right.$-axis). The plotted values in panel (A) are the fractional change in $c^{*}$ resulting from a fractional change in the demography kernel near a given size-to-size transition; these are the sum of the fractional changes in $c^{*}$ resulting from separate fractional changes in the survival/growth and fecundity components of the kernel. Darker shading indicate higher $c^{*}$ elasticities, and contour lines and values represent $c^{*}$ elasticity isoclines. (B) The elasticity function is integrated vertically to give stacked survival and reproduction elasticity values as a function of plant size in year $t, z_{1}$. (C) Similarly, the elasticity kernel is integrated horizontally to give the summed elasticity values of individuals that grow to reach a particular plant size $\left(z_{2}\right)$ in year $t+1$, stacked separately for survivors and new seedlings. The $c^{*}$ elasticity values of the SIPM 100 were rescaled to add up to 1 [by multiplying them by $\ln (\rho)$ ], allowing for direct comparison with $\lambda$ elasticity values. (D) The difference between $c^{*}$ elasticity and $\lambda$ elasticity values. Size is the natural log of rosette area. Dotted lines indicate boundaries between the small, medium, and large classes in previously published $4 \times 4$ matrix models. 


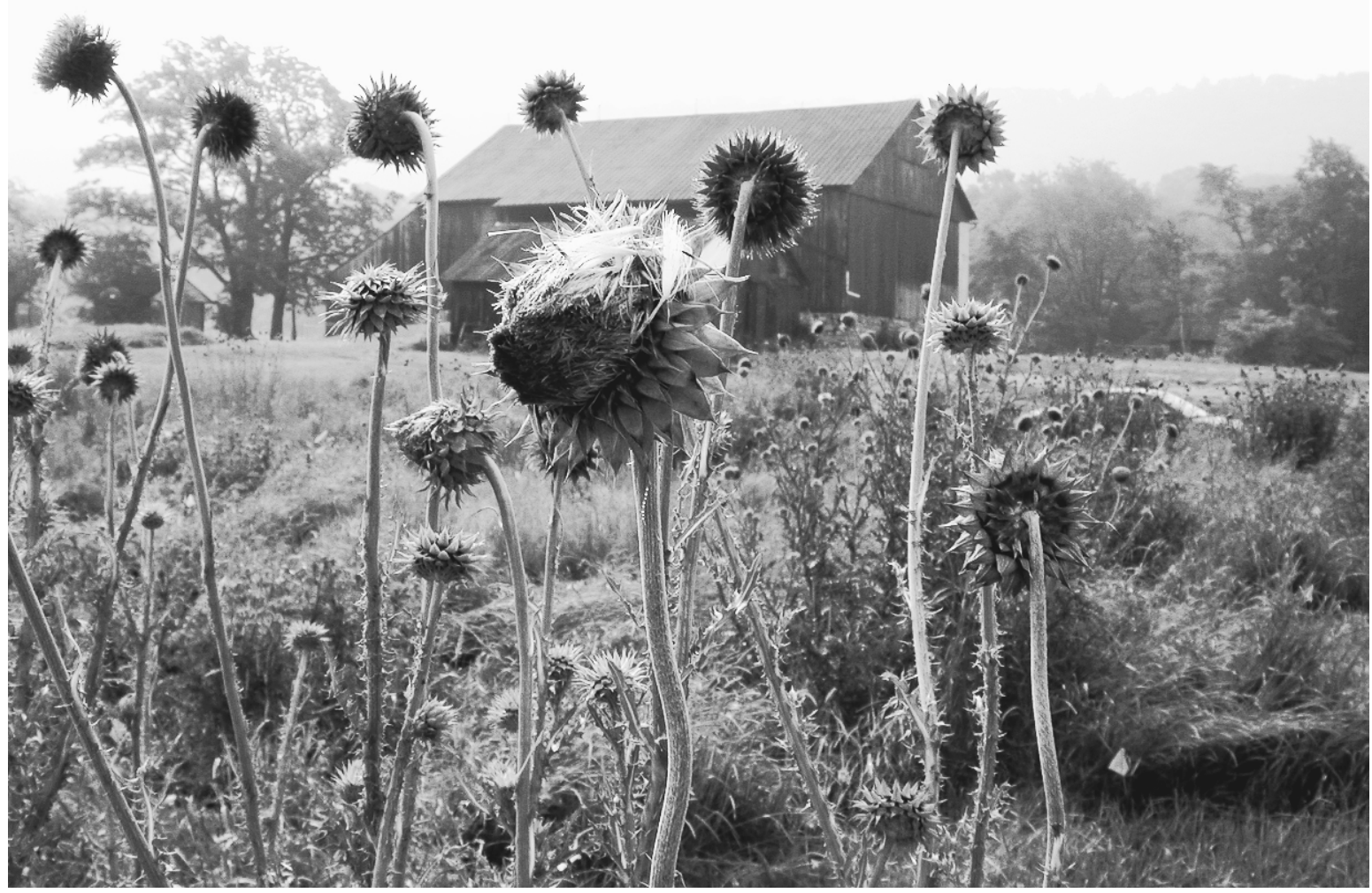

Plate 1. Carduus nutans at the Russell E. Larson Agricultural Research Farm at Rock Springs, Pennsylvania, USA. Photo credit: K. Shea.

with the population models that were previously used to study invasion speed of this species (Skarpaas and Shea 2007, Jongejans et al. 2008). SIPM and IPM elasticity analyses will therefore inform management in much greater detail (e.g., Rose et al. 2005) because they show with much higher resolution which plant sizes provide crucial management targets. Furthermore, SIPMs make more efficient use of data (especially when data are relatively sparse) and require fewer fitted parameters than would a matrix model of the required complexity (Ellner and Rees 2006).

\section{... but are SIPMs always necessary?}

Still, SIPMs are less familiar than their standard matrix counterparts, and NCMs may therefore be more appealing for simple modeling questions or applications (Godfray and Waage 1991, Shea 1996). How dissimilar small NCMs are from SIPMs may depend on the species life history. In the case of $C$. nutans, $\lambda$ and $c^{*}$ stabilized at low SIPM matrix dimensions (from $7 \times 7$ upward; Fig. $2 \mathrm{~A}, \mathrm{~B})$. This quick convergence is probably related to the high and variable growth rates relative to the range of plant sizes (Fig. 1B); in tropical trees, with very slow growth rates, convergence of $\lambda$ only started at IPM dimensions of $1000 \times 1000$ (Zuidema et al. 2010). However, the finding that SIPM evaluations with small matrices perform reasonably well does not mean that matrix models of the same size automatically represent the same population dynamics. The previously published $4 \times 4 \mathrm{NCM}$ projected $20 \%$ higher $\lambda$ and $c^{*}$ estimates for $C$. nutans than the $4 \times 4$ SIPM evaluations based on the same data (Fig. 2), indicating that the choice of parameterization methods and class boundaries do matter. Comparing $c^{*}$ and $\lambda$ elasticities showed that management aimed at controlling spread should focus more on reducing the rapid, annual life cycle loop of establishing seedlings that grow to become small reproducing plants, whereas management aimed at controlling local population densities should focus relatively more on plants that survive and grow for one or more years and then produce many seeds (Fig. 4D). Interestingly, a standard $4 \times 4 \mathrm{NCM}$ also picks up the relatively higher $c^{*}$ elasticity for the small-to-small transition, but not the reduced elasticity values of intermediate-sized survivors, because those are still in the "small" rosette size range in the $4 \times 4$ NCM. Such elasticity differences can be decisive for the choice of management strategies (Shea et al. 2010). In short, 
SIPMs presumably reflect actual population dynamics better than NCMs, although explicitly testing model predictions relative to data is beyond the scope of this study, and merits future research. Rules of thumb for when the heuristically appealing matrix models are adequate would be very valuable.

\section{Future challenges}

Future prospects for improving the realism and accuracy of analytical population models involve incorporating temporal variation within years and seasons (Ellner and Rees 2007, Rees and Ellner 2009), because annual matrix models are likely to miss dynamics at shorter time scales. This seems particularly important in the case of density-dependent seasonal vital rates. One approach for including small timescales is a periodic matrix model, which requires the identification and parameterization of seasonal vital rates (Caswell and Trevisan 1994, Picó et al. 2002, Schleuning et al. 2008). Another approach is to include time as a continuous variable (Méndez et al. 2009), although such models require considerable sub-annual demographic data. As with the individual variation discussion, there is also a need for further investigation of when temporal model complexity is a necessity, vs. when annual models are good enough for analyzing population dynamics and for assisting management decisions.

Our finding that invasion speed is highly sensitive to variation in dispersal parameters is consistent with other studies (Bullock et al. 2008, Muller-Landau et al. 2008, Soons and Bullock 2008). In particular, variation in wind speed had a high $c^{*}$ elasticity value in our study (Fig. 3). This indicates that the exciting trend of systematically incorporating the effects of environmental drivers on demographic processes (Bakker et al. 2009) needs to be extended to include environmental effects on dispersal. Formally including environmental drivers in population models has the additional advantage of enabling the comparison of direct and indirect pathways through which the environment affects spread rates and the analysis of selection pressures on plant traits in different environments (Jongejans et al. 2010). Such modeling approaches not only will show under which circumstances spatial spread rates are more sensitive to changes in dispersal or demography, but also will reveal how much model parameters are able to vary, given observed or predicted climate fluctuations or given management options. The spatial integral projection models introduced in this paper are a step toward increasingly complex population models that are, at same time, analytically tractable.

\section{ACKNOWLEDGMENTS}

We are grateful to Jessica Metcalf and two anonymous reviewers for helpful comments. This research was funded by the National Science Foundation (grant DEB-0614065 to K. Shea) and the Netherlands Organisation for Scientific Research (NWO-veni grant 863.08.006 to E. Jongejans).

\section{Literature Cited}

Bakker, V. J., D. F. Doak, G. W. Roemer, D. K. Garcelon, T. J. Coonan, S. A. Morrison, C. Lynch, K. Ralls, and R. Shaw. 2009. Incorporating ecological drivers and uncertainty into a demographic population viability analysis for the island fox. Ecological Monographs 79:77-108.

Bullock, J. M., R. F. Pywell, and S. J. Coulson-Phillips. 2008. Managing plant population spread: prediction and analysis using a simple model. Ecological Applications 18:945-953.

Burnside, O. C., C. R. Fenster, L. L. Evetts, and R. F. Mumm. 1981. Germination of exhumed weed seed in Nebraska. Weed Science 29:577-586.

Caswell, H. 2001. Matrix population models. Construction, analysis, and interpretation. Second edition. Sinauer, Sunderland, Massachusetts, USA.

Caswell, H., and M. C. Trevisan. 1994. Sensitivity analysis of periodic matrix models. Ecology 75:1299-1303.

Childs, D. Z., M. Rees, K. E. Rose, P. J. Grubb, and S. P. Ellner. 2003. Evolution of complex flowering strategies: an age- and size-structured integral projection model. Proceedings of the Royal Society of London B 270:1829-1838.

DeAngelis, D. L., and L. J. Gross. 1992. Individual-based models and approaches in ecology: populations, communities and ecosystems. Chapman and Hall, New York, New York, USA.

de Kroon, H., J. M. van Groenendael, and J. Ehrlén. 2000. Elasticities: a review of methods and model limitations. Ecology 81:607-618.

Easterling, M. R., S. P. Ellner, and P. M. Dixon. 2000. Sizespecific sensitivity: applying a new structured population model. Ecology 81:694-708.

Ellner, S. P., and M. Rees. 2006. Integral projection models for species with complex demography. American Naturalist 167: $410-428$.

Ellner, S. P., and M. Rees. 2007. Stochastic stable population growth in integral projection models: theory and application. Journal of Mathematical Biology 54:227-256.

Enright, N. J., M. Franco, and J. Silvertown. 1995. Comparing plant life histories using elasticity analysis: the importance of life span and the number of life-cycle stages. Oecologia 104: $79-84$.

Evans, M., N. Hastings, and B. Peacock. 1993. Statistical distributions. Second edition. John Wiley, New York, New York, USA.

Godfray, H. C. J., and M. Rees. 2002. Population growth rates: issues and an application. Philosophical Transactions of the Royal Society of London B 357:1307-1319.

Godfray, H. C. J., and J. K. Waage. 1991. Predictive modelling in biological control: the mango mealy bug (Rastrococcus invadens) and its parasitoids. Journal of Applied Ecology 28: 434-453.

Jongejans, E., H. Huber, and H. de Kroon. 2010. Scaling up phenotypic plasticity with hierarchical population models. Evolutionary Ecology 24:585-599.

Jongejans, E., K. Shea, O. Skarpaas, D. Kelly, A. W. Sheppard, and T. L. Woodburn. 2008. Dispersal and demography contributions to population spread of Carduus nutans in its native and invaded ranges. Journal of Ecology 96:687-697.

Judson, O. P. 1994. The rise of the individual-based model in ecology. Trends in Ecology and Evolution 9:9-14.

Katul, G. G., A. Porporato, R. Nathan, M. Siqueira, M. B. Soons, D. Poggi, H. S. Horn, and S. A. Levin. 2005. Mechanistic analytical models for long-distance seed dispersal by wind. American Naturalist 166:368-381.

Kelly, D., and K. McCallum. 1990. Demography, seed biology and biological control of Carduus nutans in New Zealand. Pages 72-79 in J. P. Palmer, editor. The biology and control of invasive plants. Proceedings of a conference of the British Ecological Society, Cardiff, Wales.

Kuss, P., M. Rees, H. H. Aegisdottir, S. P. Ellner, and J. Stocklin. 2008. Evolutionary demography of long-lived monocarpic perennials: a time-lagged integral projection model. Journal of Ecology 96:821-832. 
Lewis, M. A., M. G. Neubert, H. Caswell, J. S. Clark, and K. Shea. 2006. A guide to calculating discrete-time invasion rates from data. Pages 169-192 in M. W. Cadotte, S. M. McMahon, and T. Fukami, editors. Conceptual ecology and invasion biology: reciprocal approaches to nature. Springer, Dordrecht, The Netherlands.

Méndez, V., D. Campos, and A. W. Sheppard. 2009. A model for plant invasions: the role of distributed generation times. Bulletin of Mathematical Biology 71:1727-1744.

Metcalf, J. C., K. E. Rose, and M. Rees. 2003. Evolutionary demography of monocarpic perennials. Trends in Ecology and Evolution 18:471-480.

Morris, W. F., and D. F. Doak. 2004. Buffering of life histories against environmental stochasticity: accounting for a spurious correlation between the variabilities of vital rates and their contributions to fitness. American Naturalist 163:579590 .

Muller-Landau, H. C., S. J. Wright, O. Calderon, R. Condit, and S. P. Hubbell. 2008. Interspecific variation in primary seed dispersal in a tropical forest. Journal of Ecology 96:653667.

Neubert, M. G., and H. Caswell. 2000. Demography and dispersal: calculation and sensitivity analysis of invasion speed for structured populations. Ecology 81:1613-1628.

Neubert, M. G., and I. M. Parker. 2004. Projecting rates of spread for invasive species. Risk Analysis 24:817-831.

Okubo, A., A. Hastings, and T. Powell. 2001. Population dynamics in temporal and spatial domains. Pages 298-373 in A. Okubo and S. A. Levin, editors. Diffusion and ecological problems: modern perspectives. Springer, New York, New York, USA.

Pfister, C. A., and F. R. Stevens. 2003. Individual variation and environmental stochasticity: implications for matrix model predictions. Ecology 84:496-510.

Picó, F. X., H. de Kroon, and J. Retana. 2002. An extended flowering and fruiting season has few demographic effects in a Mediterranean perennial herb. Ecology 83:1991-2004.

Popay, A. I., and R. W. Medd. 1990. The biology of Australian weeds 21. Carduus nutans L. ssp. nutans. Plant Protection Quarterly 5:3-13.

R Development Core Team. 2009. R: A language and environment for statistical computing. $\mathrm{R}$ Foundation for Statistical Computing, Vienna, Austria.

Rees, M., D. Z. Childs, J. C. Metcalf, K. E. Rose, A. W. Sheppard, and P. J. Grubb. 2006. Seed dormancy and delayed flowering in monocarpic plants: selective interactions in a stochastic environment. American Naturalist 168:E53E71.

Rees, M., and S. P. Ellner. 2009. Integral projection models for populations in temporally varying environments. Ecological Monographs 79:575-594.
Rees, M., and K. E. Rose. 2002. Evolution of flowering strategies in Oenothera glazioviana: an integral projection model approach. Proceedings of the Royal Society of London B 269:1509-1515.

Rose, K. E., S. M. Louda, and M. Rees. 2005. Demographic and evolutionary impacts of native and invasive insect herbivores on Cirsium canescens. Ecology 86:453-465.

Schleuning, M., V. Huaman, and D. Matthies. 2008. Flooding and canopy dynamics shape the demography of a clonal Amazon understorey herb. Journal of Ecology 96:1045-1055.

Shea, K. 1996. Estimating the impact of control efforts: models of population dynamics. Plant Protection Quarterly 11(Supplement 2):263-265.

Shea, K. 2004. Models for improving the targeting and implementation of biological control of weeds. Weed Technology 18:1578-1581.

Shea, K., E. Jongejans, O. Skarpaas, D. Kelly, and A. Sheppard. 2010. Optimal management strategies to control local population growth or population spread may not be the same. Ecological Applications 20:1148-1161.

Shea, K., and D. Kelly. 1998. Estimating biocontrol agent impact with matrix models: Carduus nutans in New Zealand. Ecological Applications 8:824-832.

Shea, K., A. Sheppard, and T. Woodburn. 2006. Seasonal lifehistory models for the integrated management of the invasive weed nodding thistle Carduus nutans in Australia. Journal of Applied Ecology 43:517-526.

Skarpaas, O., and K. Shea. 2007. Dispersal patterns, dispersal mechanisms and invasion wave speeds for invasive thistles. American Naturalist 170:421-430.

Skellam, J. G. 1951. Random dispersal in theoretical populations. Biometrika 38:433-435.

Skinner, K., L. Smith, and P. Rice. 2000. Using noxious weed lists to prioritize targets for developing weed management strategies. Weed Science 48:640-644.

Soons, M. B., and J. M. Bullock. 2008. Non-random seed abscission, long-distance wind dispersal and plant migration rates. Journal of Ecology 96:581-590.

Williams, J. L., and E. E. Crone. 2006. The impact of invasive grasses on the population growth of Anemone patens, a longlived native forb. Ecology 87:3200-3208.

Zuidema, P. A., and M. Franco. 2001. Integrating vital rate variability into perturbation analysis: an evaluation for matrix population models of six plant species. Journal of Ecology 89:995-1005.

Zuidema, P. A., E. Jongejans, P. D. Chien, H. J. During, and F. Schieving. 2010. Integral Projection Models for trees: a new parameterization method and a validation of model output. Journal of Ecology 98:345-355.

\section{APPENDIX}

Mathematical details of spatial integral projection models (Ecological Archives E092-008-A1). 